\section{Hyperfeinstruktur des GeTe}

E. Tiemann, J. Hoeft und T. Törring

II. Physikalisches Institut der Freien Universität Berlin

(Z. Naturforsch. 26 a, 1930 [1971] ; eingegangen am 18. Oktober 1971)

In einer zusammenfassenden Darstellung ${ }^{1}$ haben wir für die Gruppe der zweiatomigen (IV/VI)-Verbindungen die Systematik der elektrischen Dipolmomente und Quadrupolkopplungskonstanten aufgezeigt. Zur Diskussion der systematischen Variation der Kopplungskonstanten von ${ }^{73} \mathrm{Ge}$ fehlt der Wert für GeTe. Der Gang dieser Größe ist von besonderem Interesse für die spätere Analyse der chemischen Bindung in dieser Molekelklasse.

Über das Mikrowellenrotationsspektrum des GeTe ist in einer früheren Arbeit ${ }^{2}$ berichtet worden. Die Empfindlichkeit des verwendeten $100 \mathrm{kHz}$-Stark-EffektSpektrometers reichte seinerzeit nicht aus, um Rotationsübergänge von isotopen Molekeln mit ${ }^{73} \mathrm{Ge}$ (Kernspin $I=9 / 2$ ) in natürlicher Häufigkeit zu messen. Die häufigste Isotopenkombination mit ${ }^{73} \mathrm{Ge}$ ist mit $2,6 \%$ natürlicher Konzentration vertreten. Daraus ergibt sich, daß die stärkste HFS-Komponente im Rotationsübergang $J=2 \rightarrow 3$ einer isotopen Molekel ohne HFS mit einer Häufigkeit von $0,6 \%$ entspricht.

Die Empfindlichkeit des Spektrometers wurde durch den Einsatz eines „Signal-Averager" und durch Phasen-Synchronisation der Strahlungsquelle verbessert. Gemittelt wurden 32 Überläufe à $10 \mathrm{~s}$. Die Zeitkonstante am Ausgang des Lock-in-Verstärkers betrug $300 \mathrm{~ms}$ bei einer Filtersteilheit von $12 \mathrm{~dB} /$ Oktave. Bei Temperaturen um $570{ }^{\circ} \mathrm{C}$ erreichten wir ein SignalRausch-Verhältnis von maximal $3: 1$.

Die Messungen wurden an den beiden Isotopenkombinationen ${ }^{73} \mathrm{Ge}^{128} \mathrm{Te}$ und ${ }^{73} \mathrm{Ge}^{130} \mathrm{Te}$ durchgeführt. Tabelle 1 enthält die Linienfrequenzen. In die Auswertung wurde die Frequenz $v_{\mathrm{s}}$ des hypothetischen Übergangs ohne HFS (siehe Tab. 1) einbezogen, die mit Hilfe der bekannten Massenrelationen ${ }^{\mathbf{3}}$ zwischen den Rotationskonstanten aus den in ${ }^{2}$ angegebenen Daten berechnet wurde. Hierfür wurden die atomaren Massen

Sonderdruckanforderungen an Dr. E. Tiemann, II. Physikalisches Institut der Freien Universität Berlin, D-1000 Berlin 33, Boltzmannstr. 20.

1 J. Hoeft, F. J. Lovas, E. Tiemann u. T. Törring, J. Chem. Phṿs. 53, 2736 [1970].
Tab. 1. Hyperfeinstruktur des Rotationsübergangs $J=2 \rightarrow 3$ von ${ }^{73} \mathrm{Ge}^{128} \mathrm{Te}$ und ${ }^{73} \mathrm{Ge}^{130} \mathrm{Te}$ im Schwingungsgrundzustand. Zahlen in ( ) : Meßfehler in Einheiten der letzten Dezimalen.

\begin{tabular}{|c|c|c|}
\hline Molekel & $F \rightarrow F^{\prime}$ & $v(\mathrm{MHz})$ \\
\hline \multirow{3}{*}{${ }^{73} \mathrm{Ge}^{128} \mathrm{Te}$} & $13 / 2 \rightarrow 15 / 2$ & $11904,254(100)$ \\
\hline & $\begin{aligned} 11 / 2 & \rightarrow 13 / 2 \\
9 / 2 & \rightarrow 9 / 2\end{aligned}$ & $11901,719(100)$ \\
\hline & $\left.\begin{array}{rl}11 / 2 & \rightarrow 9 / 2 \\
5 / 2 & \rightarrow 3 / 2\end{array}\right\}$ & $11902,639(100)$ \\
\hline
\end{tabular}

Hypothetischer Übergang $J=2 \rightarrow 3, v=0$, $v_{\mathrm{S}}=11906,043(12) \mathrm{MHz}$

$\begin{array}{rlr}{ }^{73} \mathrm{Ge}^{130} \mathrm{Te} & 13 / 2 \rightarrow 15 / 2 & 11837,601(100) \\ 11 / 2 \rightarrow 13 / 2 & 11835,094(100) \\ 9 / 2 \rightarrow 9 / 2 & & \\ 11 / 2 & \rightarrow 9 / 2 & 11835,942(100) \\ 5 / 2 \rightarrow 3 / 2 & & \end{array}$

Hypothetischer Úbergang $J=2 \rightarrow 3, v=0$, $v_{\mathrm{S}}=11839,319(12) \mathrm{MHz}$

in den Tabellen von Mattauch ${ }^{4}$ et al. verwendet. Die Berechnung der Hyperfeinenergien erfolgte nach den in ${ }^{3}$ angegebenen Formeln. In Tab. 2 ist die Kopp-

\begin{tabular}{lll}
\hline Molekel & $e q_{0} Q(\mathrm{MHz})$ \\
\hline $\mathrm{GeO}^{\text {a }}$ & 208,33 & $(\mathbf{1 9})$ \\
$\mathrm{GeS}^{\mathrm{a}}$ & 187,76 & $(\mathbf{1 2})$ \\
$\mathrm{GeSe}^{\mathrm{a}}$ & 172,40 & $(25)$ \\
$\mathrm{GeTe}^{\mathrm{N}}$ & 153,1 & $(30)$
\end{tabular}

Tab. 2. Hyperfeinstrukturkonstanten von ${ }^{73} \mathrm{Ge}$.

a Siehe Zitat ${ }^{1}$.

lungskonstante e $q_{0} Q$ angegeben, die sich aus der Mittelung über beide Isotopenkombinationen ergab. Zur Veranschaulichung des systematischen Gangs sind die Kopplungskonstanten der drei anderen Germaniumverbindungen mit angegeben.

2 J. Hoeft u. H.-P. Nolting, Z. Naturforsch. 22 a, 1121 [1967].

3 C. H. Townes u. A. L. Schawlow, Microwave Spectroscopy, McGraw-Hill Book Co., London 1955.

4 J. H. E. Mattauch, W. Thiele u. A. H. Wapstra, Nucl. Phys. 67, 1 [1965]. 